\title{
Calculating Three-Phase Relative Permeabilities Using Network Modeling
}

\author{
FENWICK, Darryl H., Stanford University \\ BLUNT, Martin J., Stanford University
}

Paper presented at the 5th European Conference on the Mathematics of Oil Recovery, Leoben, Austria, 3-6 Sept. 1996

\begin{abstract}
This paper investigates three-phase flow in water-wet porous media. Three-phase flow differs from two-phase flow due to the effects of pore-scale double displacement mechanisms and the ability for oil to exist in a continuous layer between the water and gas. These oil layers have an important impact upon the recovery of oil. Double displacement mechanisms and the effects of oil layers are incorporated into a three-dimensional network model. The network model allows macroscopic parameters such as capillary pressure, relative permeability, and oil recovery to be calculated. Several types of displacement are analyzed by specifying a series of changes in capillary pressure. Different paths in capillary pressure space result in drastically different three-phase relative permeabilities and oil recoveries. All three-phase relative permeabilities demonstrate strong hysteresis, which cannot be accounted for in current empirical models.
\end{abstract}

\section{INTRODUCTION}

The modeling of multiphase flow in porous media requires knowledge of the capillary pressure and relative permeability functions. ${ }^{1}$ It is very difficult to measure relative permeability and capillary pressure for the full range of possible three-phase saturations and saturation histories (see Oak $e t$ $a .^{2}$ for a listing of relative permeability studies up to 1990). Most numerical models of three-phase flow rely on empirical relationships between capillary pressure, saturation, and relative permeability. ${ }^{3-7}$ Baker, ${ }^{8}$ Delshad and Pope, ${ }^{9}$ and Oak ${ }^{10}$ compared empirical models to published experimental data. With only a few exceptions they showed that empirical models could not match the measured relative permeabilities.

Empirical models fail because they do not account for the physics of flow. All three-phase models assume that the capillary pressure and relative permeabilities are unique functions of saturation and independent of fluid properties. As is shown later, this is not the case and such assumptions make large errors, particularly for flow at low oil saturations.

One way of incorporating physics into the prediction of relative permeability is through pore-level modeling. To simulate the flow accurately, a proper understanding of the pore-scale displacement mechanisms is necessary. Due to the tremendous geometrical complexity of the pore space as well as the fluid-fluid interactions, this is a very difficult task which has not been fully accomplished. However, a great deal of experimental work studying two-phase flow at the pore-scale has been performed, especially for strongly water-wet systems. From extensive experimental studies in micromodels (see for instance the work of Lenormand and Zarcone ${ }^{11}$ ), the pore-level mechanisms for twophase flow are well understood. When these mechanisms are coded into a numerical model, the two-phase flow behavior for both consolidated and unconsolidated media, including capillary pressures and relative permeabilities can be predicted successfully. ${ }^{12-17}$

Pore-level processes in three-phase flow are less well understood. However, recent studies using transparent micromodels $^{18-20}$ have identified several important pore-level features of three-phase flow. One important feature of threephase flow is the ability of oil to form a layer between water and gas in the pore space, which has been observed in micromodels. ${ }^{18-20}$ This layer is the principal conduit for oil flow at low saturation. Another feature of three-phase flow is double displacement mechanisms, where one fluid displaces another which displaces a third. Øren et al. ${ }^{18}$ observed and described double drainage, where gas displaces oil which displaces water. Double drainage was shown to mobilize waterflood residual oil.

This paper describes a three-phase three-dimensional network model that incorporates the effects of oil layers and double displacement mechanisms. Previous authors have constructed three-phase network models. ${ }^{21-24}$ In this work, three-phase relative permeabilities calculated using a network model are presented. The relative permeabilities that are calculated show strong dependance on the capillary pressure and saturation history. In addition, the relative permeabilities for every phase show a functional dependance upon the saturations of two phases as well as fluid properties. Existing empirical models cannot capture the calculated behavior. Before presenting the network model and results, the model for oil layers is presented, followed by a description of double displacement mechanisms. 


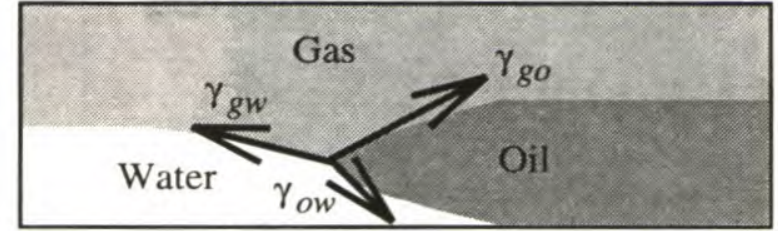

(a)

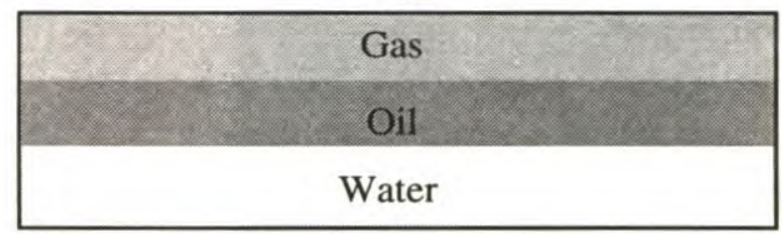

(b)

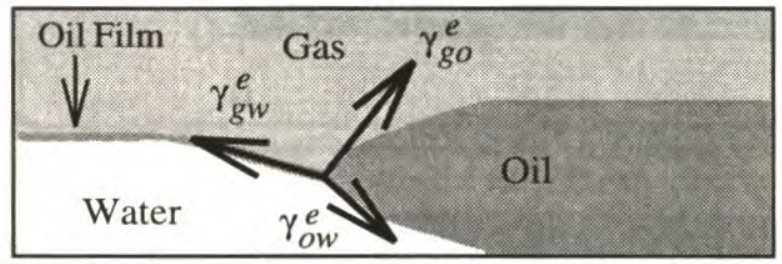

(c)

Figure 1: (a) oil initially resting on water in the presence of gas. This arrangement is only possible if $C_{s}^{i}<0$. (b) The configuration of three phases when $C_{s}^{e q}=0$. (c) The configuration when $C_{s}^{i}>0$, and $C_{s}^{e q}<0$.

\section{OIL FILMS AND LAYERS}

It has long been known that oil can spread between gas and water. ${ }^{25}$ The spreading of a oil on water in the presence of air is determined by the equation that defines the initial spreading coefficient,

$$
C_{s}^{i}=\gamma_{g w}-\left(\gamma_{o w}+\gamma_{g o}\right),
$$

where $\gamma$ is the interfacial tension, the subscript $g$ indicates a gas, $w$ indicates water, and $o$ denotes oil. Note that the interfacial tensions are for pure fluids initially in contact with each other. Fig. 1a shows that Eq. 1 represents a force balance at the three-phase contact line of water/oil/gas. If $C_{s}^{i}>0$, there is no configuration of the fluids that balances the forces in the horizontal direction. Thus, the oil spreads over the surface of the water.

When the oil is present on the gas/water interface, either through spreading or through adsorption from the vapor phase, $\gamma_{g w}$ is reduced and can be defined as an effective gas/water interfacial tension, $\gamma_{g w}^{e}$. The other interfacial tensions are also modified in the presence of a third phase (denoted $\gamma_{o w}^{e}$ and $\gamma_{g o}^{e}$ ), but it is the value of $\gamma_{g w}^{e}$ that is most dramatically affected. ${ }^{25}$ When the three phases are at thermodynamic equilibrium, the equilibrium spreading coefficient can be defined as,

$$
C_{s}^{e q}=\gamma_{g w}^{e}-\left(\gamma_{o w}^{e}+\gamma_{g o}^{e}\right) \text {. }
$$

The equilibrium spreading coefficient must be less than or equal to zero. ${ }^{26}$ If $C_{s}^{i}>0$ and $C_{s}^{e q}=0$, oil spreads as a film which will swell as more oil is added to the system (Fig. 1b). This is the case for Soltrol. ${ }^{27}$ If $C_{s}^{e q}<0$ and $C_{s}^{I}>0$, the oil will spread initially, but finite contact angles between the phases can exist such that excess oil will be found in droplets connected by thin films on the gas/water interface (Fig. 1c). This is the case for benzene ${ }^{25}$ as well as
Table 1: Reported experimental recoveries for different spreading coefficients using water-wet porous media.

\begin{tabular}{|c|c|c|}
\hline reference & \multicolumn{2}{|c|}{$S_{\text {org }}$} \\
\hline & $C_{s}^{i}>0$ & $C_{s}^{i}<0$ \\
\hline Dumoré \& Schols $^{35}$ & $2.9 \%$ & $3.2 \%$ \\
\hline Kantzas et al. $^{34}$ & $0.6-9.1 \%$ & no values \\
\hline Vizika $^{33}$ & $11 \%$ & $23 \%$ \\
\hline $\begin{array}{c}\text { Kalaydjian } \text { et al. } \\
\text { (Clashach sandstone) }\end{array}$ & $10 \%$ & $31 \%$ \\
\hline $\begin{array}{c}\text { Kalaydjian } \text { et al. } \\
\text { Ka } \\
\text { (Fountainbleu sandstone) }\end{array}$ & $16 \%$ & $20 \%$ \\
\hline Skurdal et al..$^{36}$ & $4.7 \%$ & $6.7 \%$ \\
\hline Zhou \& Blunt & \\
\hline
\end{tabular}

hexane and heptane. ${ }^{28}$ The film of oil is generally very thin, on the order of one nanometer across $\left(10^{-9} \mathrm{~m}\right){ }^{28}$

When gas contacts oil in a water-wet porous medium, the three phases behave in the same way as described above for flat films shown in Fig. 1. If $C_{s}^{i}>0$, the oil will spread between the gas and water forming a continuous film. The ability of the oil to reside between water and gas has important implications for the recovery of oil in porous media. Final oil saturations as low as $0.1 \%$ have resulted from the displacement of oil in the presence of both water and gas. ${ }^{29}$ Studies by Chatzis et al. ${ }^{30}$ Kalaydjian, ${ }^{31}$ Kalaydjian et al., ${ }^{32}$ and Vizika ${ }^{33}$ have shown a significant difference in the ultimate recovery of oil between systems with positive and negative spreading coefficient. It has been suggested that high recovery is due to the existence of films of oil between the water and gas when $C_{s}^{i}>0$, creating a continuous channel of oil through which it can drain, 30,33-35 whereas when $C_{s}^{i}<0$ such films are absent and the oil recovery is lower.

However, the simple theory that high oil recovery is due to films caused by spreading may not explain several other gravity drainage studies where similar recoveries were observed for both positive and negative $C_{s}^{i}$. Table 1 gives several references reporting residual oil saturations after gas injection $\left(S_{\text {org }}\right)$ in water-wet porous media. Note that the experiments in the references given were not identical, so direct comparisons cannot be made. The references indicate a difference in recovery between $C_{s}^{i}<0$ and $C_{s}^{i}>0$. However, the difference is sometimes very slight, and the high oil recoveries observed for $C_{s}^{i}<0$ indicate that there may be other factors involved in the recovery of oil in the presence of gas than simply the value of $C_{s}^{i}$. These seemingly contradictory studies can be resolved by realizing that thick oil layers between water and gas in crevices of the pore space can be thermodynamically favorable even for systems with $C_{s}^{i}<0$. Dong et $a l^{37}$ performed free energy calculations and found that oil can reside between water and gas in the corners of the pore space as shown in Fig. 2a for systems with $C_{s}^{i}<0$. Note that the term oil layer will be employed to distinguish oil residing in corners of the pore space, as shown for an idealized angular wedge in Fig. 2, from the thin molecular-sized oil films on a flat surface in Fig. 1. An oil layer may be several microns across, thousands of times thicker than a film. The ability of the oil to form a layer depends on the equilibrium spreading coefficient, the capillary pressures, and the angle $\beta$ of the corner. ${ }^{37}$ Calculation of oil flow rates though films show that they are very small, ${ }^{38}$ and thus the excellent oil recoveries 


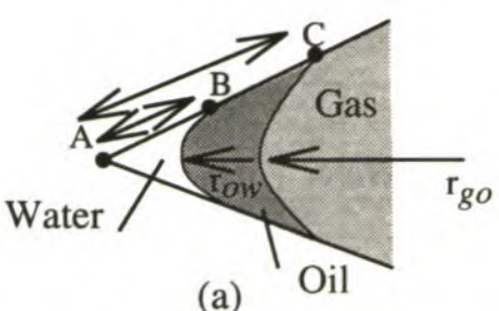

(a)

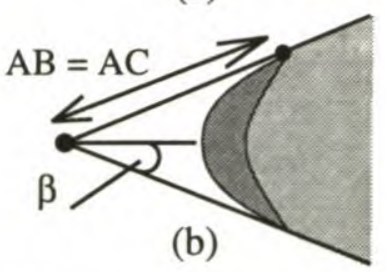

Figure 2: (a) Three phases in a corner with half angle $\beta$. (b) When the gas/oil and oil/water interfaces touch, the oil layer is assumed to be unstable.

observed experimentally must be due to drainage of much thicker oil layers in crevices in the pore space. Experimental evidence for the existence of layers in non-spreading systems was found by Hayden and Voice, ${ }^{39}$ who performed experiments on iodobenzene and found layers to exist even though $C_{s}^{i}=-8.7 \mathrm{mN} / \mathrm{m}$.

Fenwick and Blunt ${ }^{22}$ developed a simple model to capture the proposed oil layer behavior. Fig. 2 depicts three phases in a wedge found in the pore space. The fluids are assumed to behave as depicted, that is, that the solid is waterwet, the oil/water contact angle $\left(\theta_{\text {ow }}\right)$ is smaller than the gas/oil contact angle $\left(\theta_{g o}\right)$, and that the only curvature of the interface is in the plane of the wedge. Thus, using the Young-Laplace equation, ${ }^{1}$ the oil/water capillary pressure is given by $P_{\text {cow }}=\gamma_{o w} / r_{o w}$, where $r_{\text {ow }}$ is the radius of curvature of the oil/water interface, with a similar expression for the gas/oil capillary pressure $\left(P_{c g o}\right)$. The oil layer is assumed to be stable until the contact of the oil/water interface with the solid surface coincides with the gas/oil contact, as shown in Fig. $2 \mathrm{~b}$ (distance $\mathrm{AB}$ equals distance $\mathrm{AC}$ ). A ratio of interfacial curvatures can be defined as,

$$
R=\frac{r_{o w}}{r_{g o}}=\frac{P_{c g o} \gamma_{o w}}{P_{c o w} \gamma_{g o}}
$$

If $R$ is very small, then the oil layer can be very thick. The critical ratio $R_{c}$ at which the layer is no longer stable is,

$$
R_{c}=\frac{\cos \left(\theta_{\text {go }}+\beta\right)}{\cos \left(\theta_{\text {ow }}+\beta\right)}
$$

If $R<R_{c}$, then the fluid arrangement resembles Fig. 2a. If $R>R_{c}$, no oil layer is assumed to exist, and there is only a gas/water interface with an interfacial tension $\gamma_{g w}^{e}$. Although there might be a molecular-sized oil film present, this film is too thin to have any significant effect on the flow of oil. ${ }^{38}$

For a completely water-wet system it is possible to relate $\theta_{g o}$ to $C_{s}^{e q}$. Throughout this paper the system is assumed to be strongly water-wet $\left(\theta_{o w}=\theta_{g w}=0\right)$. If $\theta_{o w}=\theta_{g w}=0$,

$$
\cos \theta_{g o}=1+\frac{C_{s}^{e q}}{\gamma_{g o}},
$$

as described by Kalaydjian. ${ }^{31}$ Using Eq. 5, Eq. 4 becomes,

$$
R_{c}=1+\frac{C_{s}^{e q}}{\gamma_{g o}}-\sqrt{\left(-\frac{C_{s}^{e q}}{\gamma_{g o}}\right)\left(2+\frac{C_{s}^{e q}}{\gamma_{g o}}\right)} \tan \beta .
$$

It should be emphasized again that Eq. 6 is based upon a simplified geometric argument for oil layer stability. However, the analysis gives an indication of the expected behavior of oil layers. Layers can be present even for negative spreading coefficients, but as the spreading coefficient decreases, oil layers become increasingly less likely to exist. Notice that whether or not a oil layer exists depends on the value of $R$ which is governed by the ratio of capillary pressures, and on $R_{c}$ which depends on $C_{s}^{e q}$. Thus oil layers may be present for $C_{s}^{e q}<0$, and absent for $C_{s}^{e q}=0$, depending on the capillary pressures.

\section{DISPLACEMENT MECHANISMS}

Structure of the Porous Medium A network model represents a porous medium as a network of narrow chambers (called throats) that intersect at larger void spaces (called pores). The pore space is assumed to contain connected roughness, grooves or crevices that act as conduits for the wetting fluid, even if the center of the pore or throat is filled with non-wetting fluid. The implication is that the wetting phase is never trapped and can reach a low saturation given enough time. Experiments by Dullien ${ }^{40}$ have shown that in strongly water-wet media, wetting phase saturations as low as $1 \%$ are possible. Note that if oil layers are stable, the oil is always connected and and can never be trapped. This would appear to be a reasonable approximation, since as mentioned previously, oil saturations as low as $0.1 \%$ have been achieved (see Table 1).

Two-Phase Mechanisms Two-phase displacement mechanisms have been described most extensively by Lenormand et al. ${ }^{41}$ and Lenormand and Zarcone. ${ }^{11}$ When a non-wetting fluid displaces a wetting fluid, the process is called drainage. Drainage is characterized by piston-like displacement. At every stage in the displacement the non-wetting fluid invades the pore or throat with the lowest threshold capillary pressure. However, the non-wetting fluid may only fill a pore or throat that is adjacent to an already filled region of the pore space. The critical capillary pressure $\left(P_{c}^{*}\right)$ for the invading phase to enter a pore or throat of inscribed radius $r$ with contact angle $\theta$ is approximately,

$$
P_{c}^{*}=\frac{2 \gamma \cos \theta}{r} .
$$

If the difference in pressure between the non-wetting and wetting phases is larger than $2 \gamma \cos \theta / r$, displacement occurs.

Imbibition occurs when a wetting fluid invades a nonwetting fluid. Here the wetting fluid fills a pore or throat with the highest threshold capillary pressure. Since the wetting phase is assumed to be connected through layers in the corners and grooves throughout the pore space, the wetting fluid is able to displace non-wetting fluid in every pore or throat. The wetting layers can be either water or oil layers (if they are present). There are two types of displacement. The first is snap-off. ${ }^{42}$ For a throat of square cross-section and inscribed radius $r, P_{c}^{*}$ is ${ }^{11}$

$$
P_{c}^{*}=\frac{\gamma(\cos \theta-\sin \theta)}{r} \text {. }
$$


The second type of displacement is piston-like invasion. For a given throat piston-like invasion has a higher capillary pressure (Eq. 7) than snap-off, and so is favored, but can only occur if the adjacent pore or throat is already completely filled with wetting phase. The critical pressure for piston-like pore filling depends on the number of adjacent throats that are completely filled with wetting fluid. This is discussed in detail by Lenormand and Zarcone. ${ }^{11}$ They described the piston-like displacements as $I_{n}$, where $n$ pore throats are filled with non-wetting fluid. The displacement is most favored for small $n$. The capillary pressure for the $I_{1}$ displacement for a pore of inscribed radius $r$ is

$$
P_{c}^{*}=\frac{C_{I_{1}} \gamma}{r}
$$

where $C_{I_{1}}$ is a constant slightly less than 2 , depending on the pore geometry, the contact angle, and the number of adjacent throats next to the pore. For the $I_{2}$ displacement, the constant $C_{I_{2}}$ will be smaller than $C_{I_{1}}$. Since pores are always larger than their adjacent throats, piston-like advance in pores is always favored over snap-off. In this work, $C_{I_{1}}=$ 1.8 and $C_{I_{2}}=1.25$. $I_{n}$ displacements for $n \geq 3$ are not allowed. The non-wetting phase may be trapped if it is completely surrounded by wetting fluid.

Three-Phase Mechanisms When three phases are present in the pore space, a new series of displacements are possible, called double displacements, where one fluid displaces another which displaces the third. For a double displacement to be possible, the first and third phase must be continuous in the pore space. Øren et al. ${ }^{18}$ observed several gas $\rightarrow$ oil $\rightarrow$ water displacements in a micromodel (the $\rightarrow$ can be replaced by the word "displaces"), and called this displacement sequence double drainage. Double drainage can mobilize residual (trapped) oil and contributes to the reduction of residual oil saturation in the presence of gas and water. The gas and water must be continuous for double drainage to occur. The water is assumed to be strongly wetting and always continuous. The critical gas/water capillary pressure $P_{c g w}^{*}$ for the double drainage displacement is the summation of the capillary pressures of the individual twophase displacements. Thus, $P_{c g w}^{*}$ is

$$
P_{c g w}^{*}=2 \gamma_{g o} \cos \theta_{g o} / r_{t g o}+2 \gamma_{o w} / r_{t o w},
$$

where $r_{\text {tgo }}$ and $r_{\text {tow }}$ are the critical radii of curvature for the gas/oil and oil/water interfaces, respectively. If $P_{c g w}$ is above this value, then double drainage can occur.

The converse of double drainage is double imbibition (water $\rightarrow$ oil $\rightarrow$ gas), illustrated in Fig. 3. Note again that for double imbibition to be possible, the gas must be continuous. $P_{c g w}^{*}$ required for the displacement in Fig. 3 to occur is given by $P_{c g w}^{*}=P_{c o w}^{*}+P_{c g o}^{*}$, or,

$$
P_{c g w}^{*}=C_{I_{1}} \gamma_{o w} / r_{p o}+2 \gamma_{g o} \cos \theta_{g o} / r_{t g},
$$

where the first term is for $I_{1}$ in a pore (Eq. 9) and the second term is for piston-like advance (Eq. 7). Since this is an imbibition process, $P_{c g w}$ must be below the critical value for the displacement to occur.

By considering all possible double displacements, it is easy to show that six double displacements are possible. The critical capillary pressure for any double displacement to occur is found by summing the two-phase capillary pressures.

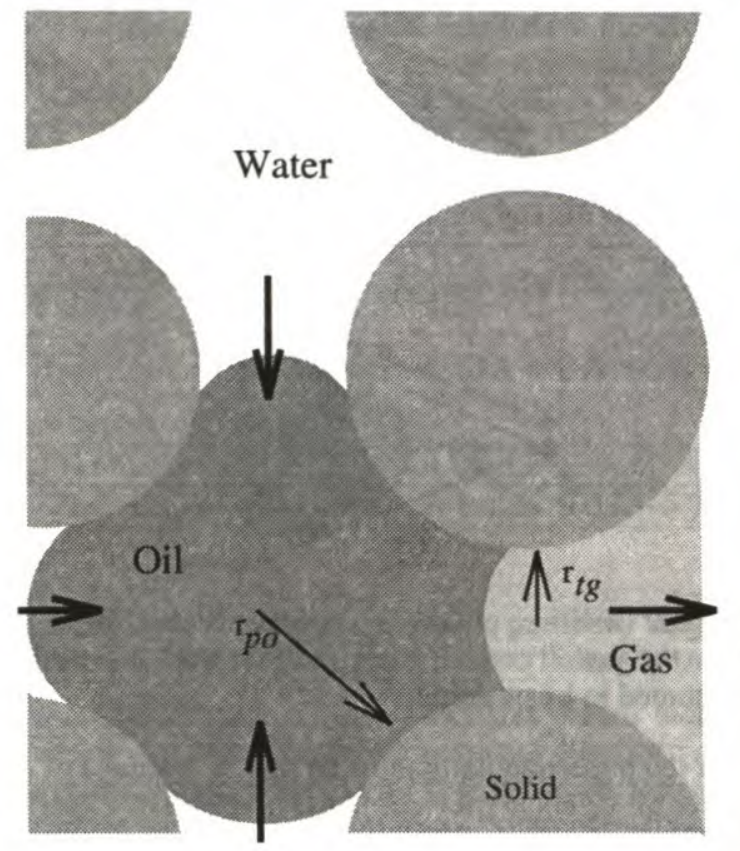

Figure 3: A schematic double imbibition displacement, where water displaces oil out of a pore by $I_{1}$ displacement. The oil subsequently displaces gas by piston-like displacement.

\section{NETWORK MODELING}

The network model is a conceptual representation of a porous medium. Pore throats connect larger pore bodies to form a network that contains an inlet and an outlet face. The network model presented here has a cubic lattice with a coordination number of six, which means that six pore throats are connected to each pore. Most porous media have average coordination numbers between four and eight. ${ }^{15}$

Periodic boundary conditions are used in the directions perpendicular to the inlet and outlet direction. The periodic boundary conditions help to minimize finite size effects in the results. For the model presented here, the pores and throats have a constant square cross-section with sides twice the length of the inscribed radius. The pores are assumed to be cubes.

The pore and throat size distributions for the inscribed radius were determined by using a Weibull distribution. ${ }^{44}$ To obtain the inscribed radius for a throat, a random number $x \in(0,1)$ is drawn, and the radius $r$ is obtained by,

$$
r=\beta(-\ln (1-x))^{\frac{1}{\alpha}} .
$$

Eq. 12 is also used to find the the lengths of the throats and aspect ratios for the pores. Table 2 gives the values for $\alpha$ and $\beta$ used for each distribution for the simulations presented. The inscribed radius for each pore is found by multiplying the aspect ratio by the average size of the 6 connecting throat radii. If that value is smaller than the largest connecting throat, the pore is set to the size of that throat. The distributions used were not taken from an actual porous medium, but are consistent with pore size distributions investigated in other work. ${ }^{15}$

How the Network Model Simulates Fluid Invasion The network model performs displacements based upon maintaining capillary equilibrium between the phases. Displacements are performed one pore or throat at a time. Each 
Table 2: Parameters used to calculate pore and throat size dimensions.

\begin{tabular}{|c|c|c|}
\hline & $\alpha$ & $\beta$ \\
\hline aspect ratio & 7 & 2 \\
\hline throat radius & 4 & $10(\mu \mathrm{m})$ \\
\hline throat length & 4 & $20(\mu \mathrm{m})$ \\
\hline
\end{tabular}

available pore or throat that can be invaded by a given phase has a $P_{c}^{*}$ associated with it that corresponds to a particular type of displacement. When a phase is selected to invade into the network, the pores and throats that are invaded are filled in order of increasing phase pressure. Thus, in drainage, the largest accessible pore or throat corresponding to the smallest capillary pressure is invaded first. In imbibition, the smallest pore or throat corresponding to the largest capillary pressure is invaded first. For example, for gas invasion into water and disconnected oil ganglia, the displacement associated with the smallest gas pressure (smallest $\left.P_{c g w}\right)$ is performed. This could be either a double drainage or gas $\rightarrow$ water displacement.

Explicit in this rule-based displacement process is the assumption that capillary forces control the displacement at the pore scale. This is not a limiting assumption, since most three-phase displacements occur at very small velocities away from wellbores.

How Saturations are Calculated The phases are all assumed to be incompressible. The pore and throat radii define the $P_{c}^{* \text { 's }}$ for displacement. Previous work with network models has found that the radii that define $P_{c}^{*}$ do not correlate directly with the volume of the pore or throat. ${ }^{14,17}$ The total volume for a throat or pore is given by,

$$
V_{t}=V_{o t} r_{t}^{\nu}, \quad V_{p}=V_{o p} r_{p}^{\mu},
$$

where $r_{t}$ is the inscribed throat radius, $r_{p}$ the inscribed pore radius, and $V_{o t}$ and $V_{o p}$ are volume prefactors. Eq. 13 is used for this work, with $V_{o t}=V_{o p}=1$ and $\nu=\mu=1.5$.

When two or three phases occupy a pore or throat, the wetting phase (water) resides in the corners of the pore space Assuming that the cross-sectional shape of pores and throats is square, the area occupied by water in the corners with contact angle $\theta$ with the solid is,

$$
A_{w}=4 r_{w}^{2}\left((\cos \theta-\sin \theta)+\theta-\frac{\pi}{4}\right),
$$

where $r_{w}$ is the interfacial radius of curvature between the water and the contacting non-wetting fluid given by $r_{w}=$ $\gamma / P_{c}$. The water volume in the corner of a pore is given by,

$$
V_{w}=\frac{A_{w}}{4 r_{p}^{2}} V_{p}
$$

with a similar expression for throats. Note that the interface is assumed to be curved in only one direction. If two phases reside in the pore or throat, the non-wetting phase volume is taken by subtracting $V_{w}$ from $V_{t}$ or $V_{p}$ as appropriate.

If three phases reside in the pore or throat with oil in a layer, the calculation for the water volume is the same, with $r_{w}=r_{o w}$ and $r_{o w}=\gamma_{o w} / P_{\text {cow }}$. Assuming again a square-shaped cross-section, the area of a oil layer in a pore or throat is,

$$
\begin{aligned}
A_{\circ}= & r_{g o}^{2}\left(\operatorname { c o s } ( \theta _ { g o } ^ { \prime } + \frac { \pi } { 4 } ) \left(\cos \left(\theta_{g \circ}+\frac{\pi}{4}\right)+\sin \left(\theta_{g o}\right.\right.\right. \\
& \left.\left.\left.+\frac{\pi}{4}\right)\right)-\frac{\pi}{4}+\theta_{g o}\right)-r_{o w}^{2}\left(1-\frac{\pi}{4}\right)
\end{aligned}
$$

where $r_{g o}=\gamma_{g o} / P_{c_{g o}}$ and $r_{o w}=\gamma_{o w} / P_{c_{o w}}$. The volume of the oil layer in the pore or throat is given by Eq. 15 with subscript $w$ replaced by $o$. The gas volume is calculated by subtracting the water and oil volumes from the total volume of the pore.

As the capillary pressure changes, the radius of curvature between the continuous phases changes. If the nonwetting phase (oil or gas) in the pore or throat is trapped, the radius of curvature is constant and is determined by the capillary pressure between the phases when the non-wetting phase was trapped. To calculate the saturation in the network model, the phase volumes in each pore and throat are found using Eq. 13-16. Thus, given the volumes of each phase at the current capillary pressures, the saturation is determined by dividing the volume of each phase by the total pore volume of the network.

How Relative Permeability is Calculated Note again that the phases are assumed to be incompressible, so for each pore $i$ the equation,

$$
\sum_{j}^{Z} q_{i j}^{\alpha}=0,
$$

is obeyed, where $q_{i j}^{\alpha}$ is the volumetric flow rate of phase $\alpha$ between pore $i$ and neighboring pores $j$. The value $Z$ is the coordination number of pore $i$. The flow rate $q_{i j}^{\alpha}$ is defined as,

$$
q_{i j}^{\alpha}=g_{i j}^{\alpha}\left(P_{i}^{\alpha}-P_{j}^{\alpha}\right),
$$

where $g_{i j}^{\alpha}$ is the conductance of phase $\alpha$ between the pores $i$ and $j$. The conductance $g_{i j}^{\alpha}$ is the harmonic mean of the conductances between the centers of pores $i$ and $j$,

$$
\frac{1}{g_{i j}^{\alpha}}=\frac{1}{g_{t}^{\alpha}}+\frac{1}{2}\left(\frac{1}{g_{i}^{\alpha}}+\frac{1}{g_{j}^{\alpha}}\right),
$$

where $g_{i}^{\alpha}$ is the conductance of phase $\alpha$ in pore $i, g_{j}^{\alpha}$ is the conductance in pore $j$, and $g_{t}^{\alpha}$ is the conductance of the connecting throat.

Conductance of the Phases The conductance of a pore or throat completely filled by one phase is assumed to be given by Poiseuille's Law, ${ }^{45}$

$$
g=\frac{\pi r_{e f f}^{4}}{8 \mu_{\alpha} l}
$$

where $r_{\text {eff }}=(\sqrt{A / \pi}+r) / 2, \mu$ is the viscosity of the phase, $A$ is the cross-sectional area of the pore or throat ( $A=4 r^{2}$ for a square cross-section), $r$ is the largest inscribed radius of the pore or throat, and $l$ is the length of the pore or throat ( $l=2 r$ for a pore).

For two phases in a pore, the conductance of the wetting phase in the corners of the pore space (i.e. the water in Fig. 2) is approximated by an expression from Ransohoff and Radke. ${ }^{46}$

$$
g^{w}=\frac{A_{w} r_{o w}^{2}}{R_{f} \mu_{w} l}
$$

where $A_{w}$ is the cross-sectional area of the water, $r_{w}$ is the interfacial curvature between the water and the non-wetting fluid, and $R_{f}$ is a dimensionless resistance factor that has been tabulated. ${ }^{46}$ The conductance of the non-wetting phase 
in the center of the throat is given by Eq. 20, except that the effective radius $r_{\text {eff }}=\left(\sqrt{A_{n w} / \pi}+r\right) / 2$, where $A_{n w}$ is the cross-sectional area of non-wetting fluid in the pore or throat.

The value of $R_{f}$ depends on the boundary condition at the fluid interface and the contact angle. An analytical expression ${ }^{47}$ is used to determine $R_{f}$ that approximates well the tabulated values determined numerically by Ransohoff and Radke. ${ }^{46}$ If the interface is a no-slip surface, $R_{f}=361$ for a square throat and a zero contact angle. This condition is a reasonable approximation for crude oil/water systems. However, when clean fluids or gas contact the wetting phase in the corner, the conditions may not be best approximated by a no slip condition. If a no stress boundary condition at the interface is assumed, $R_{f}=113$. This is appropriate for gas/water and gas/oil interfaces.

If there is no slip or partial slip at the fluid interface and the interface is mobile, then the flow of one phase affects the others. This leads to cross-terms in the relative permeabilities, where the pressure gradient in one phase can cause flow in another. For two-phase flow, this issue has been discussed by Kalaydjian. ${ }^{48}$ In this work all interfaces are assumed to be no stress boundaries.

For three phases existing in a pore or throat, the conductances for the water and gas are given by Eq. 21 and Eq. 20 respectively, and $r_{e f f}=\left(\sqrt{A_{g} / \pi}+r\right) / 2$. The conductance of an oil layer between water and gas is given by,

$$
g^{o}=\frac{A_{o} r_{g o}^{2}}{R_{f} \mu_{o} l}
$$

where $R_{f}$ is determined by an analytical function that accounts for the oil/water interfacial curvature and the boundary conditions at the two interfaces. ${ }^{47}$

Calculating the Relative Permeability The relative permeability of a given phase in the network model is calculated by selecting a section of the network model away from the inlet to avoid boundary effects. Here, the last half of a $30 \times 15 \times 15$ network model (flow is in the $x$-direction) is chosen. The saturation for the section of the network is calculated. The conductance of each pore and throat for each phase is determined as described above. The absolute permeability is determined once using the single phase expression for conductance in pores and throats. The conductance between pores is found using Eq. 19. Eq. 17 is written for each pore using Eq. 18, with constant pressure boundary conditions on each side of the section of the network. This generates a set of equations with a matrix that contains the conductances between the pores. The pressures are the unknown values, which are determined using an iterative conugate gradient solver. Given the pressures in the pores, the flow rates for the phases in and out of the section of the network can be determined using Eq. 18. Given the total flow into or out of the section of the network, the phase viscosity, the imposed pressure drop, and the absolute permeability, the multiphase Darcy's law is applied to determine the relative permeability of the phase at the calculated saturation.

Note that if a phase is not connected from one side of the $15 \times 15 \times 15$ network to another, its relative permeability is zero. Water is always connected, but gas and oil can be trapped.

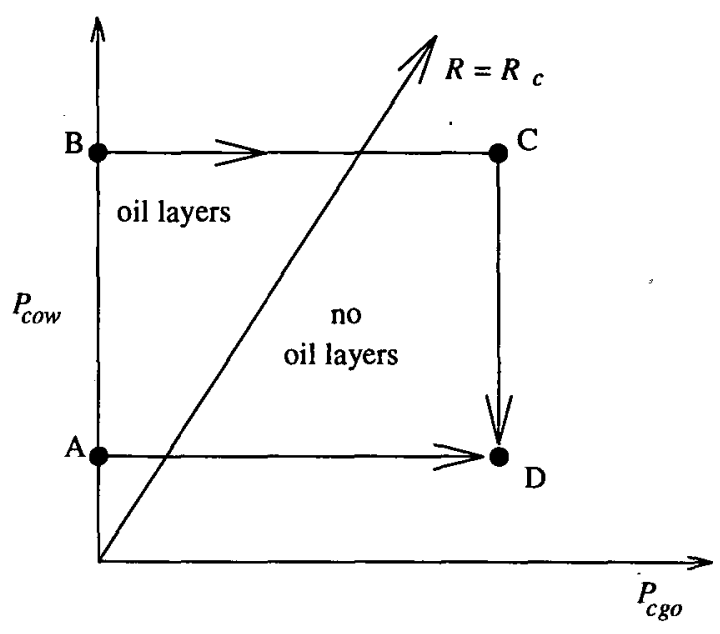

Figure 4: Schematic diagram representing different displacements as routes in capillary pressure space.

\section{Three-Phase Relative Permeability and Hysteresis}

Routes in Capillary Pressure Space Fig. 4 is a schematic diagram representing different three-phase displacements as paths in $P_{c g o}-P_{\text {cow }}$ space. All oil/water two-phase displacements occur on the $P_{\text {cow }}$ axis. When gas is introduced into the system, $P_{c g o}$ is defined and finite. The line denoted by $R=R_{c}$ separates the region where oil layers are stable from the region where they do not exist. In this work, two different displacements representing paths BCD and AD are examined. Let B in Fig. 4 represent a two-phase oil/water system at a high capillary pressure corresponding to high oil saturation. If gas invades into the system, eventually, point $C$ will be reached, and a certain amount of oil will be displaced. The $\mathrm{BC}$ path might have a large portion of threephase flow occurring in the region where oil layers are stable. Since the oil cannot be trapped when layers exist, the $\mathrm{BC}$ path may result in very high recovery. If water is injected into the system, three-phase imbibition occurs, reducing $P_{\text {cow }}$ to point $\mathrm{D}$. This imbibition process may also result in increased oil recovery due to double imbibition displacements.

If the system is at point $\mathrm{B}$ and water is injected, $P_{\text {cow }}$ can be lowered to A, where the oil is found only in disconnected ganglia. If gas is injected into the oil and water, the majority of the displacement may occur in the region where oil layers are not stable, and the disconnected oil could only be displaced through double drainage.

At point D, the capillary pressures for both sequences of displacements would be the same. Yet before any actual results are calculated, one can already imagine that the residual oil saturations and the relative permeabilities can be drastically different. Results are presented for two different cases, one where $C_{s}^{e q}=0$, representing a system where layers are most likely to exist such as air/Soltrol/water, another where $C_{s}^{e q}=-4 m N / m$, representing a system such as air/n-decane/water. ${ }^{28}$

Results The network initially contains $100 \%$ water. Primary drainage to $90 \%$ oil saturation is performed. Two different displacements are then investigated. The first is gas injection into $90 \%$ oil, followed by imbibition of water. For the second displacement, water is injected into the network, displacing oil until the residual oil saturation is reached $\left(S_{o r}=30 \%\right.$ ). Subsequently, gas invades into the network. 


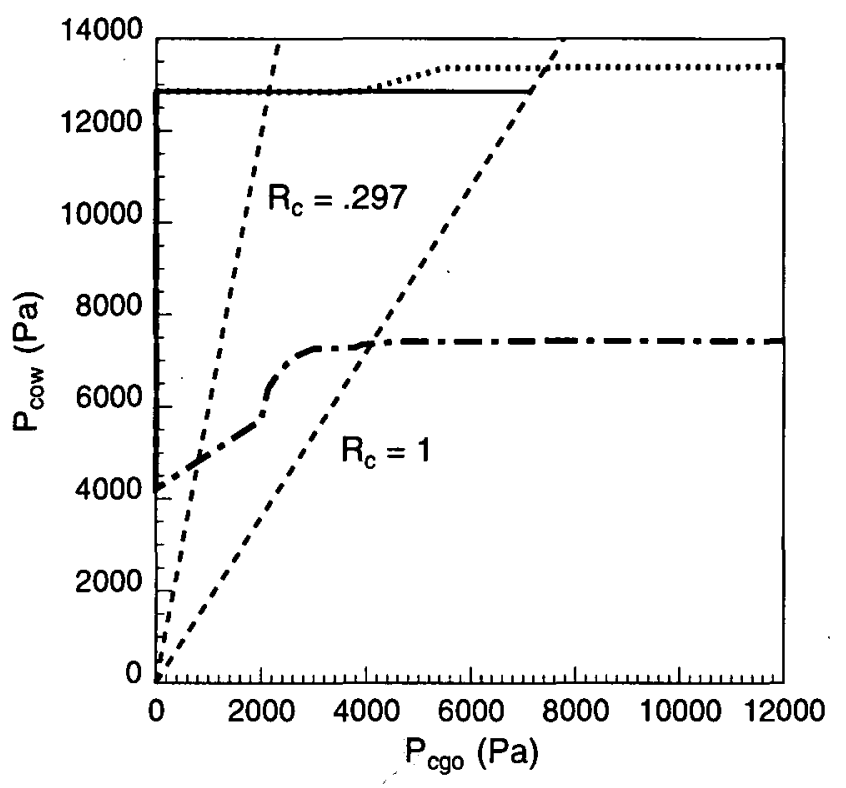

- gas inj. into $90 \%$ oil, $\mathrm{C}_{\mathrm{s}}^{\mathrm{eq}}=0$ ......... gas inj. into $90 \%$ oil, $\mathrm{C}_{\mathrm{s}}^{\mathrm{eq}}=-4 \mathrm{mN} / \mathrm{m}$ - - gas injl. into res. oil

Figure 5: Capillary pressure pathways for the two different displacements. The dashed lines indicated by $R_{c}=1$ and $R_{c}=0.297$ separate the regions where oil layers are present from the regions where layers do not exist. For $C_{s}^{e q}=0$, from Eq. 6, $R_{c}=1$. For $C_{s}^{e q}=-4 m N / m$, from Eq. 6, $R_{c}=0.297$.

For gas injection into residual oil, only the displacement for $C_{s}^{e q}=-4 m N / m$ is shown. The results for the displacement with $C_{s}^{e q}=0$ are similar.

Fig. 5 shows the capillary pressure paths for the various displacements. The solid line in Fig. 5 represents path BC for $C_{s}^{e q}=0$. Note that the entire displacement occurs in the region where oil layers are stable. The dotted line represents path BC for $C_{s}^{e q}=-4 \mathrm{mN} / \mathrm{m}$. The displacement continues off the scale, including the displacement representing path $\mathrm{CD}$. Note that a large portion of the displacement occurs in the region where oil layers do not exist. Similarly for the dash-dot line representing path AD. Fig. 6 shows the saturation pathways that correspond to the capillary saturation pathways in Fig. 5. When $C_{s}^{e q}=0$, gas invasion into $90 \%$ oil results in $100 \%$ recovery. Clearly, this is the ideal case. Before the gas enters the network, the oil is continuous. When gas is introduced into the system, oil layers are stable and thus the oil stays continuous. For $C_{s}^{e q}=0$, the point at which oil layers disappear $\left(R=R_{c}=1\right.$ from Eq. 6) also corresponds to the $P_{c g o}$ necessary for the gas to enter the smallest oil-filled throat. Thus the gas can displace all of the oil while oil layers are stable, resulting in $100 \%$ recovery. Note than no water is displaced due to the gas preferentially displacing oil.

When $C_{s}^{e q}=-4 m N / m$, path BC no longer recovers all the oil. $45 \%$ of the oil remains trapped by both gas and water, which is a poorer recovery than by waterflooding. This is caused by the disappearance of oil layers early in the displacement ( $R_{c}=0.297$ from Eq. 6). When gas invades oil and oil layers do not exist, it is unable to form loops in the pore space. When gas attempts to connect two gas-

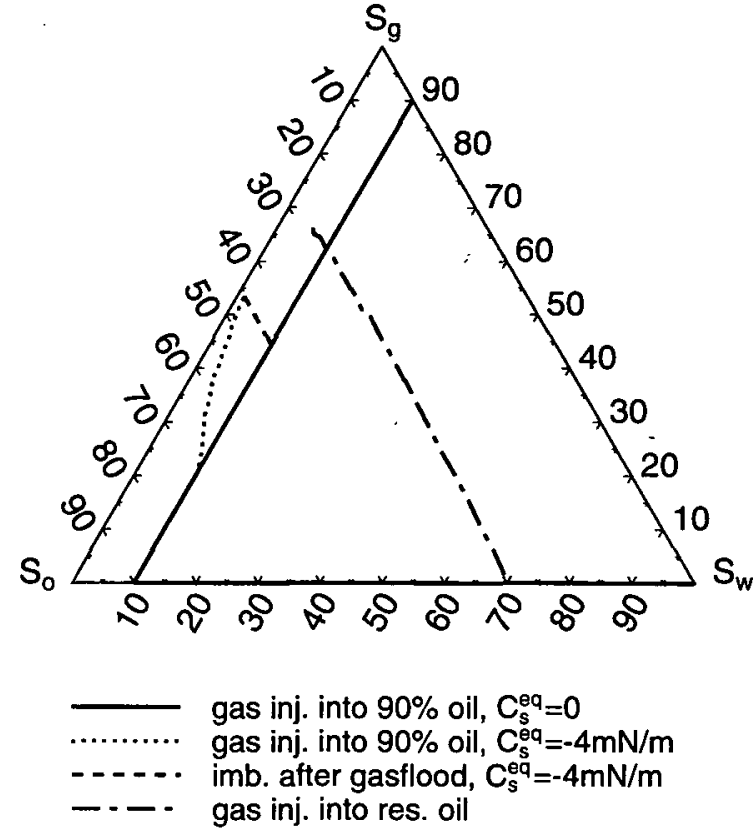

Figure 6: Ternary diagram indicating saturation pathways for the displacements shown in Fig. 5.

filled portions of the network, it must first displace a disconnected region of oil. This is impossible, and thus the gas is forced to maintain a poorly connected dendritic structure. Gas can only form loops by displacing water. This phenomenon leads to significant trapping of oil in both pores and throats. This issue is discussed for two-phase flow in more detail by Blunt et al. ${ }^{12}$ After gas injection, water invades the system (path $C D$ ) and $P_{c o w}$ is reduced (off the scale in Fig. 5). The gas rapidly becomes disconnected by snap-off with an increase of $S_{w}$ of $8.5 \%$. Double imbibition does occur, but in this example it does not displace a significant amount oil.

The AD path is represented by gas injection into waterflood residual oil, which is $30 \%$ for this case. For this displacement, oil layers become unstable at a much lower $P_{c g o}$, before any significant amount of oil is displaced. $P_{\text {cow }}$ is defined as the lowest pressure before the oil is disconnected during imbibition. When gas is injected, double drainage reconnects the oil briefly. $P_{\text {cow }}$ then rises, as shown. The incremental oil recovery, which is merely $3 \%$ in this case, is due solely to double drainage unaided by oil layer flow. The final residual oil saturation of $27 \%$ occurs when $P_{c g o}$ reaches over $110 \mathrm{kPa}$, which is off the scale in Fig. 5 .

Relative Permeabilities The three-phase relative permeabilities for the displacements described above are shown in Fig. 7. All the relative permeability curves show significant hysteresis. For gas injection into $90 \%$ oil, $k_{r o}$ for both $C_{s}^{e q}=0$ and $C_{s}^{e q}=-4 \mathrm{mN} / \mathrm{m}$ is similar at high oil saturations. However, when $S_{o}$ is lowered, the $k_{r o}$ 's deviate due to the effect of layers. For $C_{s}^{e q}=0$, the relative permeability is finite down to $S_{o}=0$. The $k_{r o}$ at low $S_{o}$ is due to flow in oil layers. Note the constant slope on the log-log plot for saturations less than $30 \%$, where $k_{\text {ro }}$ is controled solely by the conductance of oil layers. For $C_{s}^{e q}=-4$ $m N / m$, oil layers are not stable, resulting in significant of trapping. When gas invades into residual oil, oil layers are 
not stable regardless of the value for $C_{s}^{e q}$, and the oil may be trapped. $k_{r o}$ starts and ends at zero, but is finite when double drainage reconnects the oil.

The gas relative permeabilities in Fig. 7 are a function of $C_{s}^{e q}, S_{g}, S_{o}$, and capillary pressure history. For $C_{s}^{e q}=0$, $k_{r g}$ for gas invasion into $90 \%$ oil saturation is similar to the non-wetting curve in primary drainage. This is because gas is non-wetting and oil and water combined behave as the wetting phase. When gas is invading into residual oil and water, the oil does not appear to be completely wetting in the presence of gas, and thus $k_{r g}$ is different for this case. When gas invades into $90 \%$ oil saturation for $C_{s}^{e q}=-4 m \mathrm{~m} / \mathrm{m}$, layers are not stable, and again oil is not completely wetting to gas. However, since the trapped oil saturation is different from the other two cases, $k_{r g}$ is not the same as for gas injection into $90 \%$ oil. Water imbibition after gasflood causes rapid reduction in the gas relative permeability due to snapoff.

The water relative permeability curves for gas invasion into residual oil and water is similar to a two-phase $k_{r w}$. When gas invasion into $90 \%$ oil is examined, for $C_{s}^{e q}=0$ the saturation remains constant, thus $k_{r w}$ is constant throughout the displacement at a value of $2.7 \times 10^{-4}$. For $C_{s}^{e q}=$ $-4 m N / m$, it is apparent that $k_{r w}$ depends on saturation history. At low water saturation, the water resides principally in wetting layers in the corners of the pore space. For $C_{s}^{e q}<0$, the oil/water and gas/water interfaces in the corner have different radii of curvature, and hence a oil-filled throat has a different water saturation and conductance than a gas-filled throat. The overall water conductance is thus a function of both the oil and gas saturations.

\section{DISCUSSION}

The pore level configuration and displacement of three phases based on considerations of capillary equilibrium and observations from micromodel experiments have been studied. This analysis allows for the construction of a rule-based three-phase network model.

The relative permeabilities, especially for flow at low oil saturation, are sensitive to saturation pathway and the equilibrium spreading coefficient. Existing empirical models cannot capture this relative permeability behavior.

\section{CONCLUSIONS}

Oil layers between water and gas in the pore space can exist in systems with a negative initial spreading coefficient. An approximate expression has been developed for when such layers are stable. These layers allow oil to remain connected and to flow at very low saturation.

Three-phase flow allows a series of six double displacement mechanisms where one phase displaces another that displaces a third. Double drainage and double imbibition are mechanisms that can mobilize previously trapped oil.

Network modeling allows for the prediction from physical principles the oil recovery, relative permeabilities and capillary pressures for a variety of different displacements.

Three-phase relative permeabilities and capillary pressures are strong functions of saturation history and fluid properties and cannot be described adequately by any of the available empirical models. The relative permeabilities for all phases exhibited hysteresis.
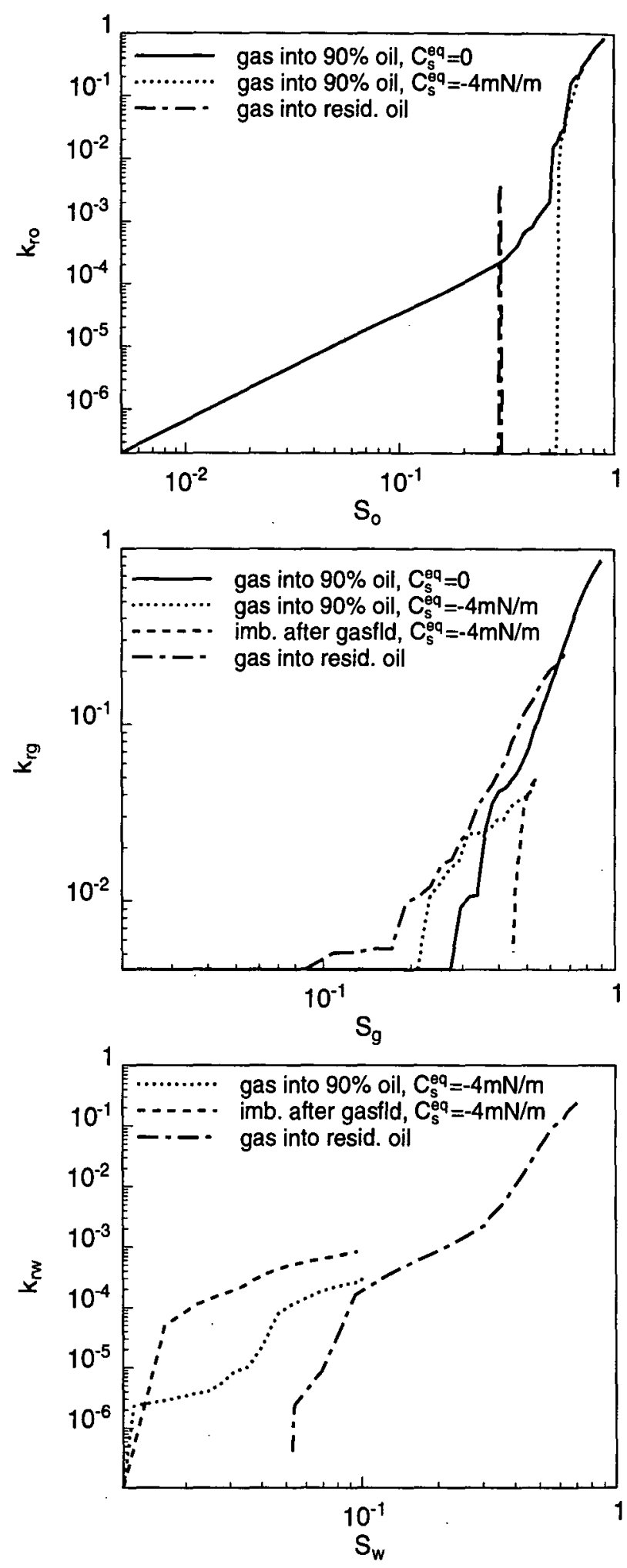

Figure 7: Three-phase relative permeabilities for the displacements shown in Fig. 6. 


\section{REFERENCES}

1. Dullien, F. A. L. Porous Media: Fluid Transport and Pore Structure. Academic Press, San Diego, second edition, 1992.

2. Oak, M. J., Baker, L. E., and Thomas, D. C. Threephase relative permeability of berea sandstone. Journal of Petroleum Technology, 42:1057-1061, August 1990.

3. Fayers, F.J. Extension of stone's method 1 and conditions for real characteristics in three-phase flow. Society of Petroleum Engineers Reservoir Engineering, 4:437-445, 1989.

4. Parker, J.C., Lenhard, R.J., and Kuppusamy, T. A parametric model for constitutive properties governing multiphase flow in porous media. Water Resources Research, 23:618$624,1987$.

5. Stone, H. L. Probability model for estimating three-phase relative permeability. Journal of Petroleum Technology, 20:214-218, February 1970.

6. Stone, H. L. Estimation of three-phase relative permeability and residual data. Journal of Canadian Petroleum Technology, 12:53-61, 1973.

7. van Genuchten, M. T. A closed-form equation for predicting the hydraulic conductivity of unsaturated soils. Soil Science Society of American Journal, 44:892-898, 1980.

8. Baker, L. E. Three-phase relative permeability correlations, paper SPE 17369 proceedings of the 1988 Sixth SPE/DOE Symposium on Enhanced Oil Recovery, Tulsa, OK, April.

9. Delshad, M., and Pope, G. A. Comparison of the threephase oil relative permeability models. Transport in Porous Media, 4:59-83, 1989.

10. Oak, M. J. Three-phase relative permeability of water-wet berea, paper SPE 20183 proceedings of the 1990 Seventh SPE/DOE Symposium on Enhanced Oil Recovery, Tulsa, OK, April.

11. Lenormand, R. and Zarcone, C. Role of roughness and edges during imbibition in square capillaries, paper SPE 13264 proceedings of the 1984 SPE Annual Technical Conference and Exhibition, Houston, TX, September.

12. Blunt, M., King, M.J. and Scher, H. Simulation and theory of two-phase flow in porous media. Physical Review A, 46:7680-7699, December 1992.

13. Bryant, S. and Blunt, M. J. Prediction of relative permeability in simple porous media. Physical Review A, 46:20042011, August 1992.

14. Heiba, A. A., Sahimi, M., Scriven, L.E., and Davis, H. T. Percolation theory of two-phase relative permeability. SPE Reservoir Engineering, 7:123-132, February 1992.

15. Jerauld, G.R. and Salter, S.J. The effect of pore-structure on hysteresis in relative permeabilty and capillary pressure: Pore-level modeling. Transport in Porous Media, 5:103$151,1990$.

16. Lenormand, R., Touboul E., and Zarcone, C. Numerical models and experiments on immiscible displacements in porous media. Journal of Fluid Mechanics, 189:165, 1988.

17. McDougall, S.R. and Sorbie, K.S. The impact of wettability on waterflooding: Pore-scale simulation. SPE Reservoir Engineering, 10:208-213, August 1995.

18. Øren, P.E., Billiote, J., and Pinczewski, W.V. Mobilization of waterflood residual oil by gas injection for water-wet conditions. SPE Formation Evaluation, 7:70-78, March 1992.

19. Øren, P.E. and Pinczewski, W.V. The effect of wettability and spreading coefficients on the recovery of waterflood residual oil by miscible gasflooding, paper SPE 24881 proceedings of the 1992 67th Annual Technical Conference and Exhibition of the SPE, Washington D.C., October.

20. Soll, W. E., Celia, M. A., and J.L. Wilson. Micromodel studies of three-fluid porous media systems: pore-scale processes relating to capillary pressure-saturation relationships. Water Resources Research, 29(9):2963-2974, 1993.

21. Soll, W. E. and Celia, M. A. A modified percolation approach to simulating three-fluid capillary pressuresaturation relationships. Advances in Water Resources, 16(2):107-126, 1993.

22. Fenwick, D. H., and Blunt, M. J. Pore level modelling of three phase flow in porous media, proceedings of the 1995 8th European Symposium on Improved Oil Recovery, Vienna, Austria, May.

23. Øren, P.E., Billiote, J., and Pinczewski, W.V. Pore-scale network modelling of waterflood residual oil recovery by immiscible gas flooding, paper SPE 27814 proceedings of the 1994 Improved Oil Recovery Symposium, Tulsa, OK, April.

24. Pereira, G.G., Pinczewski, W.V., Chan, D.Y.C., Paterson, L., and Øren, P.E. Pore-scale network model for drainage dominated three-phase flow in porous media. accepted for publication, Transport in Porous Media, 1996.

25. Adamson, A.W. Physical Chemistry of Surfaces. John Wiley \& Sons, New York, fifth edition, 1990.

26. Rowlinson, J. S., and Widom, B. Molecular Theory of Capillarity. Clarendon Press, Oxford, first edition, 1989.

27. Øren, P.E. and Pinczewski, W.V. The effect of film-flow on the mobilization of waterflood residual oil by gas flooding, proceedings of the 1991 6th European IOR Symposium, Stavanger, Norway, May.

28. Hirasaki, G.J. Structural interactions in the wetting and spreading of van der waals fluids. J. Adhesion Sci. Technol., 7(3):285-322, 1993.

29. Zhou, D., and Blunt, M. J. Distribution of light nonaqueous phase liquid in the subsurface. submitted to Journal of Contaminant Hydrology, 1995.

30. Chatzis, I., Kantzas, A., and Dullien, F.A.L. On the investigation of gravity-assisted inert gas injection using micromodels, long berea sandstone cores, and computer-assisted tomography, paper SPE 18284 proceedings of the 198863rd Annual Technical Conference and Exhibition of the SPE, Houston, TX, October.

31. Kalaydjian, F. J.-M. Performance and analysis of threephase capillary pressure curves for drainage and imbibition in porous media, paper SPE 24878 proceedings of the 1992 67th Annual Technical Conference and Exhibition of the SPE, Washington D.C., October.

32. Kalaydjian, F. J.-M., Moulu, J-C., Vizika, O., and Munkerud, P. K. Three-phase flow in water-wet porous media: Determination of gas/oil relative permeabilities under various spreading conditions, paper SPE 26671 proceedings of the 1993 68th Annual Technical Conference and Exhibition of the SPE, Houston, TX, October.

33. Vizika, $O$. Effect of the spreading coefficient on the efficiency of oil recovery with gravity drainage, proceedings of the 1993 Symposium on Enhanced Oil Recovery, presented before the Division of Petrolium Chemistry, Inc., Denver, $\mathrm{CO}$.

34. Kantzas, A., Chatzis, I., and Dullien, F.A.L. Enhanced oil recovery by inert gas injection, paper SPE 17379 proceedings of the 1988 Sixth SPE/DOE Symposium on Enhanced Oil Recovery, Tulsa, OK, April. 
35. Dumoré, J.M. and Schols, R.S. Drainage capillary pressure functions and the influence of connate water. Soc. Pet. Eng. J., 14:437-444, October 1974.

36. Skurdal, H., Hustad, O. St., and Holt, T. Oil recovery by gravity drainage during gas injection, proceedings of the 1995 8th European Symposium on Improved Oil Recovery, Vienna, Austria, May.

37. Dong, M., Dullien, F.A.L., and Chatzis, I. Imbibition of oil in film form over water present in edges of capillaries with an angular cross-section. J. Colloid \& Interface Sci., 172:278-288, December 1995.

38. Blunt, M.J., Zhou, D., and Fenwick, D.H. Three phase flow and gravity drainage in porous media. Transport in Porous Media, 20:77-103, 1995.

39. Hayden, N.J., and Voice, T.C. Microscopic observation of a napl in a three-fluid-phase soil system. Journal of Contaminant Hydrology, 12:217-226, 1993.

40. Dullien, F.A.L., Zarcone, C., MacDonald, I.F., Collins, A. and Bochard, R. D.E. The effects of surface roughness on the capillary pressure curves and the heights of capillary rise in glass bead packs. Journal of Colloid and Interface Science, 127:362-372, February 1989.

41. Lenormand, R., Zarcone, C. and Sarr, A. Mechanisms of the displacement of one fluid by another in a network of capillary ducts. Journal of Fluid Mechanics, 135:337-353, 1983.

42. Roof, J.G. Snap-off of oil droplets in water-wet pores. Soc. Pet. Eng. J., 10:85-90, March 1970.

43. Nilsen, L.S., Øren, P.E., Bakke, S., and Henriquez, A. Prediction of relative permeability and capillary pressure from a pore model, paper SPE 35531 proceedings of the 1996 European 3-D Reservoir Modelling Conference, Stavanger, Norway, April.

44. Weibull, W. A statistical distribution function of wide applicability. Journal of Applied Mechanics, 18:293-297, 1951.

45. Batchelor, G. K. An Introduction to Fluid Dynamics. Cambridge University Press, Cambridge, first edition, 1991.

46. Ransohoff, and Radke, C.J. Laminar flow of a wetting liquid along the corners of a predominantly gas-occupied noncircular pore. Journal of Colloid and Interface Science; 121:392-401, February 1988.

47. Zhou, D., Blunt, M. J., and Orr, F. M. Jr. Hydrocarbon drainage along corners of noncircular capillaries. submitted to Journal of Colloid and Interface Science, 1995.

48. Kalaydjian, F. Origin and quantification of coupling between relative permeabilities for two-phase flows in porous media. Transport in Porous Media, 5(3):215-229, 1990. 